\title{
Fetal neck tumors antenatal and intrapartum management
}

\author{
Jakub Kornacki, Jana Skrzypczak \\ Division of Reproduction, Department of Obstetrics, Gynaecology and Gynaecologic Oncology, \\ Poznan University of Medical Sciences, Poland
}

\begin{abstract}
In this review article we describe the ante- and perinatal management of fetal neck tumors. Although there are rare congenital anomalies, the clinical consequences for the fetus may be fatal and include airways obstruction, heart insufficiency, pulmonary hypoplasia and cosmetic effect. The right management allows to decrease the fetal and neonatal mortality and morbidity associated with the disease. It includes intrauterine therapy in some cases, mostly in a goitrus hypothyroidism of the fetus, but firstly, an assessment of the fetal airways patency with a subsequent, eventual Ex-Utero Intrapartum Treatment (EXIT).
\end{abstract}

Key words: fetus, neck tumors, ultrasound, airways obstruction, Ex-Utero Intrapartum Treatment

\section{INTRODUCTION}

Fetal tumors are very rare congenital anomalies. Their incidence is around 1 in 12000 to 1 in 30000 births [1]. Among them, neck tumors represent an important group. This is a very specific and unique fetal and neonatal problem. Initially, like other fetal tumors, neck masses may negatively affect the fetal cardiac function because of increased cardiac afterload and preload. Secondly, they may affect the patency of fetal airways, including trachea, which may have fatal consequences after birth. In addition, big neck tumors, especially teratomas, may cause fetal lung hypoplasia, because of wedging the lungs into the apices of chest cavity [2]. Finally, they have great cosmetic effect on the child.

A differential diagnosis of the fetal neck tumor or cystic lesions include lymphangioma, haemangioma, goiter, teratoma and neuroblastoma [2, 3].

The aim of this review is to bring together the topic of fetal neck tumors, especially the importance of their clinical antenatal and peripartum management to decrease the fetal and neonatal morbidity and mortality.

\section{ANTENATAL MANAGEMENT OF FETAL NECK TUMORS AND DIFFERENTIAL DIAGNOSIS}

The antenatal management of fetal neck tumors includes primarily the diagnosis. This can be achieved using 2D ultrasound. Some additional information may be obtained through MRI, especially if a significant part of the tumor is intracorporeal. Three dimensional (3D) ultrasound has a limited role in diagnosis and management of fetal neck tumors. For further monitoring of the mass it is very important to measure its dimensions (at least the length and the width). Both ultrasound and magnetic resonance imaging (MRI) enable the differentiation between solid or partly solid tumors such as fetal goiter (Fig. 1), teratoma (Fig. 2) or neuroblastoma and cystic or multicystic lesions like lymphangioma (Fig. 3) and hemangioma. In comparison to solid, hyperechoic and regular character of goiter, echogenicity of teratoma is usually more heterogenic with both solid and cystic components. Neck is a very rare localization of neuroblastoma. Differential diagnosis of lymphangioma and hemangioma includes an assessment of cystic content of the tumor. It is much more hyperechoic in hemangioma. An 


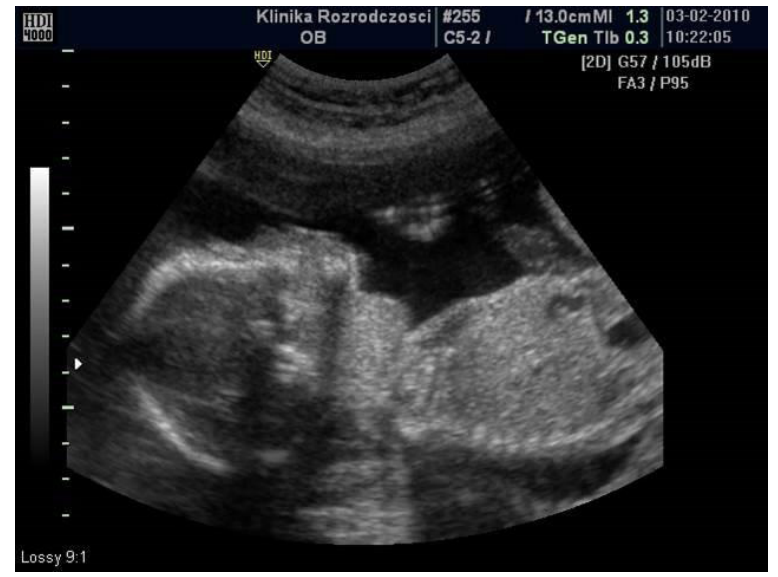

Figure 1. A longitudinal scan of a fetus with a goiter at 20 weeks gestation

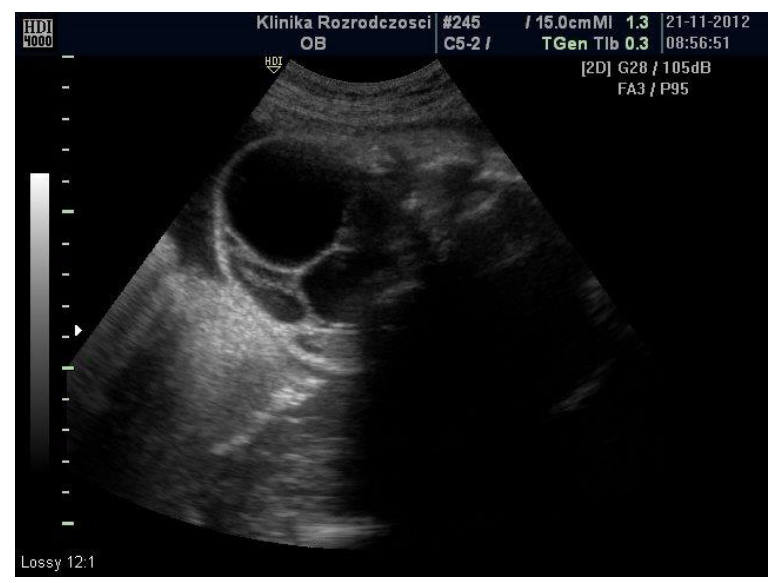

Figure 3. A fetal neck lymphangioma

ultrasound assessment of the mass should also include the vascularization of the tumor. The solid masses, like goiter, teratoma, or neuroblastoma may be highly vascularized (Fig. 4). In such a case there is an increased risk of a development of a heart failure in the fetus because of increased preload. Therefore, in all the cases of fetal neck masses, especially in solid or partly solid ones, a precise echocardiographic assessment should be done. Previously, we reported a case on a fetus with a large goiter who had to be delivered at 29 weeks due to signs of heart failure, including hydrops [3].

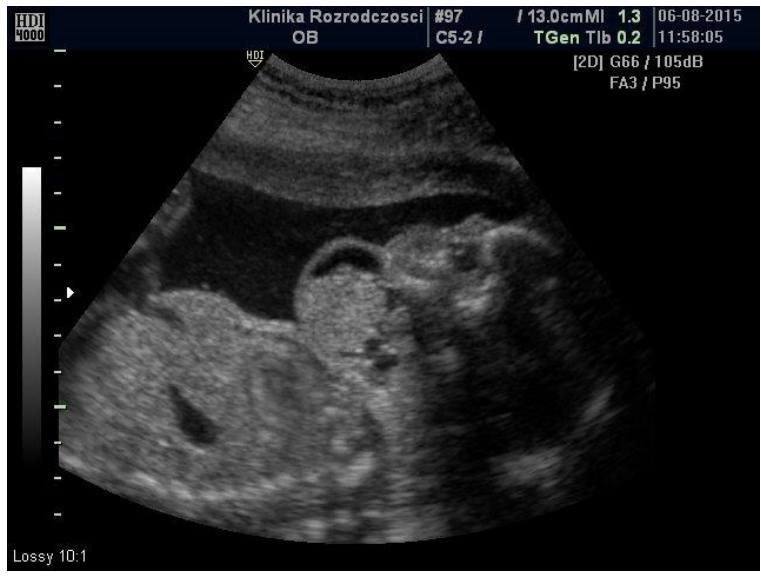

Figure 2. A longitudinal scan of a fetus with a neck teratoma

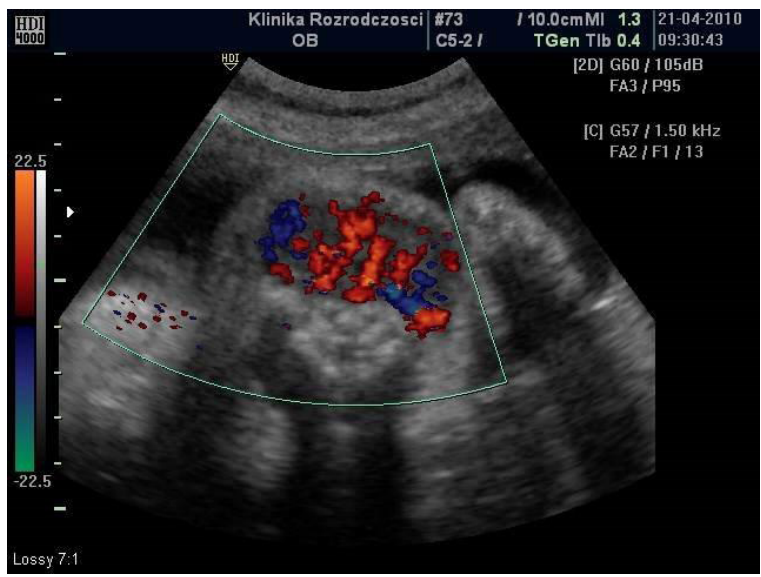

Figure 4. A transverse view of the fetus with a highly vascularized goiter

Summary of ultrasound features of different types of neck masses is presented in Table 1. Another important aspect of antenatal management of fetal neck tumors is an assessment of amniotic fluid volume. The increase of Amniotic Fluid Index (AFI) and development of polyhydramnios indicate on impaired fetal swallowing as a consequence of esophagus and trachea displacement and compression. In case of development of severe polyhydramnios causing preterm contractions and patient's dyspnea amnioreduction may be required. It may decrease the risk of preterm

Table 1. Ultrasound features of different types of fetal neck masses

\begin{tabular}{|c|c|c|c|}
\hline Type & Character & Echogenicity & Vascularization \\
\hline Goiter & Solid & Hyperechogenic & High \\
\hline Teratoma & Solid/cystic & Hyper/hypoechogenic & Variable \\
\hline Lymphangioma & Cystic & Hypoechogenic & Poor \\
\hline Hemangioma & Cystic & Hyperechogenic & High \\
\hline Neuroblastoma & Solid/cystic & Hyper/hypoechogenic & Variable \\
\hline
\end{tabular}


labor that has a direct effect on neonatal mortality and morbidity. For a fetal goiter described, we performed one amnioreduction due to AFI (Amniotic Fluid Index) of $27 \mathrm{~cm}$ and the presence of patient's dyspnea.

\section{INTRAUTERINE THERAPY}

An antenatal differential diagnosis of the fetal neck tumors may also be crucial for the further management of the fetus, including an intrauterine therapy. Mainly, this is possible for diagnosis of fetal goitrous hypothyroidism. There are several reports on the successful intrauterine treatment of goitrous hypothyroidism with repeated intraamniotic injection of L-thyroxine [3-10]. Such a treatment must follow the precise ultrasound assessment of the mass and, importantly, an amniocentesis to obtain the results of TSH, fT3 and fT4 concentrations in the amniotic fluid. The reference ranges have been established for thyroid hormones' concentrations in amniotic fluid, most recently by Singh et al. in 2003 and Baumann and Gronowski in 2007 [11, 12]. In the biggest study, described by Ribault et al., 12 fetuses were treated antenatally with intraamniotic injections of L-thyroxine for goitrous hypothyroidism [10]. In all except one of these fetuses, goiter size decrease was observed after the therapy. In the case described by us, eight intraamniotic injections of L-thyroxine were given from 23 to 37 weeks of gestation [3]. A significant reduction in goiter size was noted, as well as normalization of the TSH level in amniotic fluid, and improvement in the primarily impaired cardiac function. The above examples confirm a rather good prognosis for a diagnosis of fetal goiter. This advantageous prognosis means a reduction in goiter size with positive consequences on high airways patency and cardiac function. However, an effective intrauterine treatment of the fetal goiter does not prevent hypothyroidism after birth. Most of the affected children require further therapy with L-thyroxine $[3,10]$. The dosage of L-thyroxine usually used for the antenatal treatment are 200-800 micrograms per injection.

A second type of fetal neck tumor which may be possibly treated antenatally is a lymphangioma. The proposed method of therapy with OK-432 (sclerotherapy) injected into the neck mass in two fetuses was presented by Mikovic et al. [13]. In both cases a significant decrease in tumor size was noted. The above study is the only one describing the effective treatment of fetal neck lymphangiomas with OK-432. However, this method is widely used postnatally by pediatric surgeons. Our limited experience do not confirm the effectiveness of such therapy in case of congenital neck lymphangioma. In a similar case treated by us no significant reduction in the mass size was found despite significantly increased echogenicity of a tumor after the procedure (Fig. 5) [14].

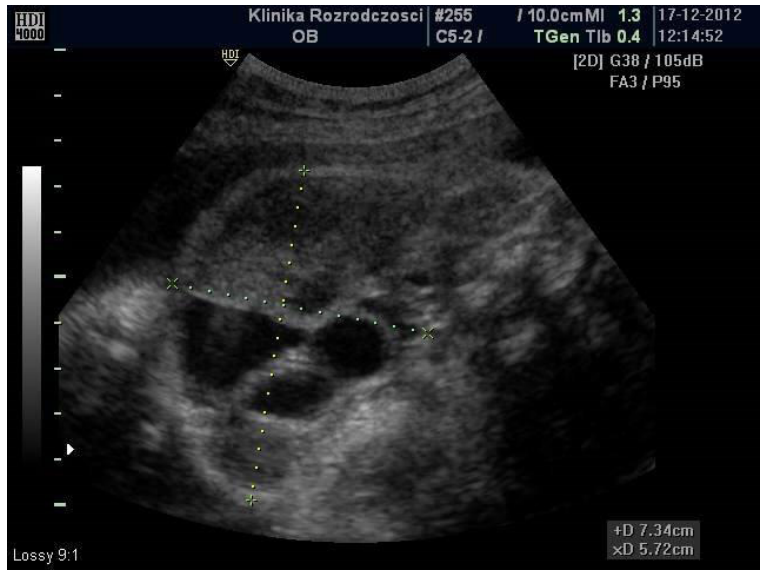

Figure 5. A fetal neck lymphangioma (3 weeks after intrauterine treatment by OK-432 injection)

The most difficult but feasible method of intrauterine therapy of fetal neck masses is open fetal tumor resection. It may be indicated in a previable fetus with signs of heart failure, including hydrops, because of increased pre- and afterload of the heart. Such procedure was successfully performed by Hirose et al. in one fetus with giant neck teratoma [15].

\section{ANTEPARTUM AND INTRAPARTUM MANAGEMENT}

Usually, the most important element of antenatal management of fetal neck tumors is a prediction of fetal airway obstruction. This can be achieved with an ultrasound, MRI or even directly by fetoscopy [16-18]. The proposed predictors of a fetal airways obstruction are: polyhydramnios, bigger size of the mass, and a presence of teratoma [16]. The additional factor predicting the fetal airway obstruction, called TEDI (tracheoesophageal displacement index) was proposed by Lazar et al. [16]. The index is defined as a sum of lateral and ventral displacements of the tracheoesophageal complex found on fetal MRI scans [16]. TEDI of $12 \mathrm{~mm}$ or greater is found to be predictive of fetal airway obstruction [16].

In each case of fetal neck tumor with suspicion of airway obstruction a caesarean section with EXIT (Ex-Utero Intrapartum Treatment) procedure should be scheduled. EXIT consists of a delivery of a head, arms and a thorax of a fetus during $\mathrm{C}$-section and a subsequent securement of the patency of fetal airways via intubation, laryngoscopy or bronchoscopy and eventually tracheostomy (Fig. 6). At the same time the placental support of the fetus is maintained. This procedure, which prevents newborn's respiratory insufficiency, significantly decreased mortality of these patients [19]. Very rarely, the tumor, especially bigger one, may be even partly resected during EXIT procedure $[15,19]$. The whole procedure, including the maintenance of placental support 


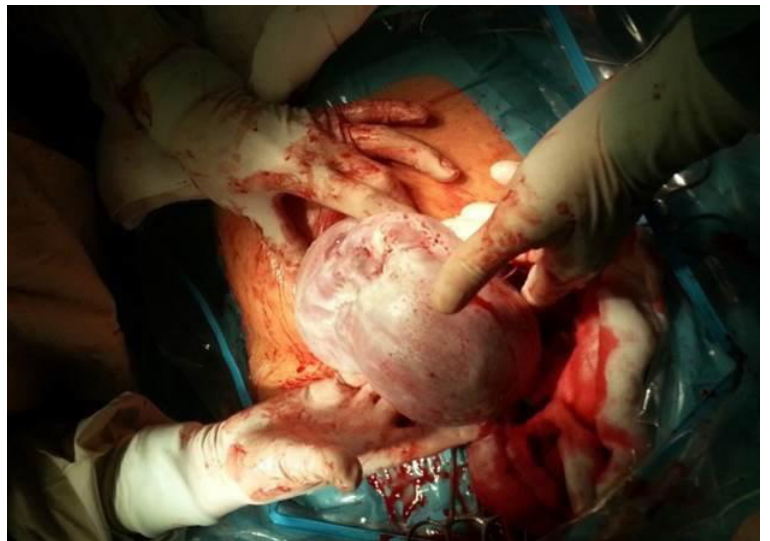

Figure 6. Delivery of a fetal head during EXIT procedure

of the fetus, requires adequate uterine muscle relaxation which is only possible through deep maternal anaesthesia $[19,20]$. During the procedure fetal life parameters should be monitored with a pulse oximeter and an intraoperative echocardiography.

The innovative, alternative method to EXIT may be fetal endoscopic tracheal intubation (FETI) [17]. The procedure consists of fetal tracheoscopy with subsequent insertion of intrauterine orotracheal cannula under ultrasound guidance. This complicated procedure ensures fetal tracheal permeability before delivery. The above procedure was described by Cruz-Martinez et al. for giant neck teratoma [17].

\section{SUMMARY}

Fetal neck tumors are very rare congenital anomalies. A differential diagnosis includes lymphangiomas, hemangiomas, teratomas, goiter and neuroblastoma. In individual cases an intrauterine therapy of masses is possible. It concerns mainly a goiter, but also may be proposed in teratoma and lymphangioma. The most important element of antenatal management of fetal neck tumors is the prediction of fetal airway obstruction. This can be achieved with ultrasound, magnetic resonance imaging (MRI), or fetoscopy/tracheoscopy. In most cases of fetal neck masses cesarean section with Ex-Utero Intrapartum Treatment (EXIT) procedure should be scheduled. It allows to avoid the most dangerous perinatal complication which is fetal/neonatal airways obstruction.

\section{Conflict of interest}

The authors declare no conflict of interest.

\section{REFERENCES}

1. Keswani SG, Crombleholme TM. Fetal tumors: clinical management. In: Kilby MD, Oepkes D, Johnson A. ed. Fetal Therapy Scientific Basis and Critical Appraisal of Clinical Benefits. Cambridge 2013: 329-340.
2. Wolfe $K$, Lewis $D$, Witte $D$, et al. Fetal cervical teratoma: What is the role of fetal MRI in predicting pulmonary hypoplasia? Fetal Diagn Ther. 2013; 33(4): 252-256, doi: 10.1159/000342191, indexed in Pubmed: 23429169.

3. Kornacki J, Mroziński B, Skrzypczak J. A rare case of recurrent fetal goiter. Fetal Diagn Ther. 2012; 31(1): 69-72, doi: 10.1159/000331454, indexed in Pubmed: 22094295.

4. Abuhamad AZ, Fisher DA, Warsof SL, et al. Antenatal diagnosis and treatment of fetal goitrous hypothyroidism: case report and review of the literature. Ultrasound Obstet Gynecol. 1995; 6(5): 368-371, doi: 10.1046/j.1469-0705.1995.06050368.x, indexed in Pubmed: 8590211.

5. Perrotin F, Sembely-Taveau C, Haddad G, et al. Prenatal diagnosis and early in utero management of fetal dyshormonogenetic goiter. Eur J Obstet Gynecol Reprod Biol. 2001; 94(2): 309-314, doi: 10.1016/s03012115(00)00346-8, indexed in Pubmed: 11165746.

6. Grüner C, Kollert A, Wildt L, et al. Intrauterine treatment of fetal goitrous hypothyroidism controlled by determination of thyroid-stimulating hormone in fetal serum. A case report and review of the literature. Fetal Diagn Ther. 2001; 16(1): 47-51, doi: 53880, indexed in Pubmed: 11125252.

7. Simsek M, Mendilcioglu I, Mihci E, et al. Prenatal diagnosis and early treatment of fetal goitrous hypothyroidism and treatment results with two-year follow-up. J Matern Fetal Neonatal Med. 2007; 20(3): 263-265, doi: 10.1080/14767050601134728, indexed in Pubmed: 17450461.

8. Mayor-Lynn KA, Rohrs HJ, Cruz AC, et al. Antenatal diagnosis and treatment of a dyshormonogenetic fetal goiter. JUltrasound Med. 2009; 28(1): 67-71, doi: 10.7863/jum.2009.28.1.67, indexed in Pubmed: 19106359.

9. Hanono A, Shah B, David R, et al. Antenatal treatment of fetal goiter: a therapeutic challenge. J Matern Fetal Neonatal Med. 2009; 22(1): 76-80, doi: 10.1080/14767050802448299, indexed in Pubmed: 19085636.

10. Ribault V, Castanet M, Bertrand AM, et al. French Fetal Goiter Study Group. Experience with intraamniotic thyroxine treatment in nonimmune fetal goitrous hypothyroidism in 12 cases. J Clin Endocrinol Metab. 2009; 94(10): 3731-3739, doi: 10.1210/jc.2008-2681, indexed in Pubmed: 19737924.

11. Singh PK, Parvin CA, Gronowski AM. Establishment of reference intervals for markers of fetal thyroid status in amniotic fluid. J Clin Endocrinol Metab. 2003; 88(9): 4175-4179, doi: 10.1210/jc.2003-030522, indexed in Pubmed: 12970283.

12. Baumann NA, Gronowski AM. Establishment of reference intervals for thyroid-stimulating hormone and free thyroxine in amniotic fluid using the Bayer ADVIA Centaur. Am J Clin Pathol. 2007; 128(1): 158-163, doi: 10.1309/69A5AV266W230AUA, indexed in Pubmed: 17580284.

13. Mikovic Z, Simic R, Egic A, et al. Intrauterine treatment of large fetal neck lymphangioma with OK-432. Fetal Diagn Ther. 2009; 26(2): 102-106, doi: 10.1159/000238111, indexed in Pubmed: 19752516.

14. Neck lymphangioma: intracystic injection of OK-432. 12th World Congress in Fetal Medicine. Marbella, Spain, June 23-27/2013.

15. Hirose $S$, Sydorak RM, Tsao $K$, et al. Spectrum of intrapartum management strategies for giant fetal cervical teratoma. J Pediatr Surg. 2003; 38(3): 446-50; discussion 446, doi: 10.1053/jpsu.2003.50077, indexed in Pubmed: 12632365.

16. Lazar DA, Cassady Cl, Olutoye OO, et al. Tracheoesophageal displacement index and predictors of airway obstruction for fetuses with neck masses. J Pediatr Surg. 2012; 47(1): 46-50, doi: 10.1016/j.jpedsurg.2011.10.022, indexed in Pubmed: 22244391.

17. Cruz-Martinez R, Moreno-Alvarez O, Garcia M, et al. Fetal Endoscopic Tracheal Intubation: A New Fetoscopic Procedure to Ensure Extrauterine Tracheal Permeability in a Case with Congenital Cervical Teratoma. Fetal Diagn Ther. 2015; 38(2): 154-158, doi: 10.1159/000362387, indexed in Pubmed: 25228387.

18. Özgünen FT, Güleç ÜK, Evrüke IC, et al. Fetal Oropharyngeal and Neck Tumors: Determination of the Need for Ex-Utero Intrapartum Treatment Procedure. Balkan Med J. 2015; 32(2): 221-225, doi: 10.5152/balkanmedj.2015.150096, indexed in Pubmed: 26167350.

19. Laje $P$, Johnson MP, Howell LJ, et al. Ex utero intrapartum treatment in the management of giant cervical teratomas. J Pediatr Surg. 2012; 47(6): 1208-1216, doi: 10.1016/j.jpedsurg.2012.03.027, indexed in Pubmed: 22703795.

20. Helfer DC, Clivatti J, Yamashita AM, et al. Anesthesia for ex utero intrapartum treatment (EXIT procedure) in fetus with prenatal diagnosis of oral and cervical malformations: case reports. Rev Bras Anestesiol. 2012; 62(3): 411-423, doi: 10.1016/S0034-7094(12)70141-1, indexed in Pubmed: 22656686. 